\title{
El incidente biopolitico. Una evaluación de la biopolítica en la obra de Michel Foucault
}

\author{
Luciano Ezequiel NOSETTO
}

Recibido: 2 de septiembre de 2011.

Aceptado: 17 de diciembre de 2011.

\section{RESUMEN}

Este trabajo propone una evaluación de la relevancia de la noción de biopolítica al interior de la obra de Michel Foucault. Para ello, se recuperan todas las ocurrencias de esta noción, a efectos de sostener su carácter de incidente. Esto implica postular a un tiempo la amplia incidencia de la noción de biopolítica al interior del recorrido foucaultiano, y el carácter incidental, episódico y secundario de este término en relación al eje de las preocupaciones del autor.

\section{PALABRAS CLAVE}

Biopoder, biopolítica, Michel Foucault.

\begin{abstract}
This article offers an evaluation of the relevance of biopolitics within Michel Foucault's work. A comprehensive analysis of Foucault's oeuvre is essential to an assessment of the various appearances of the concept of biopolitics. This makes it possible to conclude that biopolitics can be considered incidental and secondary to Foucault's main conceptual framework.
\end{abstract}

\section{KEYWORDS}

Biopower, biopolitics, Michel Foucault. 
La persistente actualidad de la obra y pensamiento de Michel Foucault (19261984) encuentra una explicación al menos parcial en la vigorosa recepción de su noción de biopolítica, presente en la bibliografía disponible ya desde mediados de la década de $1970^{1}$, pero revitalizada en tiempo reciente gracias al establecimiento y publicación de sus cursos relativos a la temática, dictados en el Collège de France ${ }^{2}$. Especialmente en el ámbito de la filosofía política italiana, la noción de biopolítica ha dado lugar a una densa articulación de nuevos diálogos conceptuales, nuevas líneas de investigación y nuevos campos de análisis políti$\mathrm{co}^{3}$. Este fenómeno de renovación conceptual y analítica ha contribuido, sin embargo, a un efecto de retroacción sobre la lectura de la obra del mismo Foucault, invitando a reorientar gran parte de su pensamiento en función de esta noción. Es en esta línea que varios autores han repuesto el pensamiento de Foucault, haciéndolo orbitar en torno al problema de la biopolítica, e indicando que, allí donde Foucault desatiende este problema, deberíamos o bien denunciar sus lagunas $^{4} \mathrm{o}$ bien sospechar su latencia ${ }^{5}$.

${ }^{1}$ La noción de biopolítica es tematizada en el capítulo 5 de La volonté de savoir, primer tomo de Histoire de la sexualité, publicado en Francia en 1976 y disponible en español desde 1977. Michel FouCAult, Histoire de la sexualité 1: La volonté de savoir, Gallimard, Paris, 1976 [Traducción al español: Historia de la sexualidad. 1- La voluntad de saber, $2^{\circ}$ edición, Siglo XXI, Buenos Aires, 2003].

${ }^{2}$ Michel Foucault, Sécurité, territoire, population. Cours au Collège de France (1977-1978), Gallimard, Paris, 2004 [Traducción al español: Seguridad, territorio, población. Curso en el Collège de France 1977-1978, Fondo de Cultura Económica, Buenos Aires, 2006]; Michel Foucault, Naissance de la biopolitique. Cours au Collège de France (1978-1979), Gallimard, Paris, 2004 [Traducción al español: Nacimiento de la biopolítica. Curso en el Collège de France 1978-1979, Fondo de Cultura Económica, Buenos Aires, 2007].

${ }^{3}$ Una mención a los aportes más salientes de la filosofía biopolítica italiana debería considerar las obras de Giorgio Agamben, Roberto Esposito y Antonio Negri. Ver, en particular, Roberto EsPosITo, Bios. Biopolítica y filosofia, Amorrortu, Buenos Aires, 2007; Giorgio AgAmBEn, Homo Sacer. El poder soberano y la nuda vida, Pretextos, Valencia, 1998; Antonio NEGRI y Michel HARDT, Imperio, Paidós, Buenos Aires, 2002. Para una presentación general y revista de esta corriente, ver Gabriel Giorgi y Fermín Rodriguez, "Prólogo", en GIORGI y RodriguEz (comps.) Ensayos sobre biopolítica. Excesos de vida, Paidós, Buenos Aires, 2007, pp. 9-34; y Ottavio MARzoccA, "Introduzione", en A.A.Vv, Lessico di biopolitica, Manifestolibri, Roma, 2006, pp. 11-20.

${ }^{4}$ Giorgio Agamben, por caso, identifica la imposibilidad de Foucault de superar las dificultades del pensamiento sobre la biopolítica, dificultades manifiestas en el hecho "de que Foucault no haya trasladado nunca su investigación a los lugares por excelencia de la biopolítica moderna: el campo de concentración y la estructura de los grandes Estados totalitarios del siglo XX." AgAmBEN, Homo Sacer. El poder soberano y la nuda vida, p. 13. Por su parte, Roberto Esposito señala en Foucault "lagunas lógicas, pequeñas incongruencias léxicas e imprevistos cambios de tono" que evidencian un "punto muerto", un "atolladero nunca superado por Foucault". La identificación del paradigma inmunitario vendría, en este sentido, a salvar a la noción de biopolítica de las incongruencias de Foucault en torno a las nociones de vida, poder y política. Esposito, Bios. Biopolítica y filosofía, pp. 53-63. 
Sin embargo, un recorrido de superficie por la obra publicada de Foucault obliga a la constatación del carácter lacunar y episódico de esta noción. Es que los términos de biopoder y biopolítica son mencionados en un número muy limitado de ocurrencias. A lo largo de su obra publicada, estos términos son presentados solo en un libro (el primer tomo de Histoire de la sexualité), en tres cursos en el Collège de France (los correspondientes al período 1976-1979) y en otras seis intervenciones, publicadas en los volúmenes de sus Dits et écrits. A esto, se suma el hecho de que, en ninguna de estas ocurrencias, el tema central es el de la biopolítica.

A efectos, entonces, de contener los problemas que una interpretación expansiva de la biopolítica podría generar en la lectura de la obra de Foucault, se propone en lo que sigue el ejercicio de suponer que la biopolítica opera a la manera de un incidente. Hablar de la incidencia de la biopolítica en el pensamiento de Foucault es decir dos cosas. Por un lado, es decir que la noción de biopolítica presenta una innovación central en el pensamiento de Foucault, con determinaciones múltiples, que implicarán no solo un desplazamiento de sus preocupaciones en adelante sino también una relectura de trabajos anteriores. Por otro lado, hablar de la incidencia de la biopolítica en el pensamiento de Foucault implica decir que la ocurrencia de la noción de biopolítica tiene el carácter de un incidente, de un episodio que otorga dinamismo a una trama, sin llegar a ser nunca una preocupación central. Amplia incidencia de la biopolítica; carácter incidental, episódico de su tratamiento: la ambivalencia del término permitirá ordenar lo que sigue.

\section{LA MEDICINA SOCIAL COMO ANTECEDENTE}

La primera ocurrencia del concepto de biopolítica en la obra publicada de Foucault corresponde a la conferencia "La naissance de la médecine sociale", dictada en la Universidad Estadual de Río de Janeiro en octubre de 19746. El objetivo de esta conferencia es el de trazar una historia de la medicalización de las

${ }^{5}$ Antonio Negri, por caso, expande la preocupación biopolítica a los últimos trabajos de Foucault indicando que allí se trama la resistencia política a partir de la vida. Antonio NegRI, Guías, Paidós, Buenos Aires, 2002, pp. 83-88; Antonio Negri, Movimientos en el Imperio, Paidós, Buenos Aires, 2006, pp. 253-260.

${ }^{6}$ Michel Foucault, "La naissance de la médecine sociale", en Daniel Defert y François Ewald (dirs.), Dits et écrits II, 1976-1988, Quattro Gallimard, Paris, 2001, pp. 207-228. 
sociedades occidentales a partir del siglo dieciocho, entendiendo por medicalización el hecho de que la existencia, la conducta, el comportamiento y el cuerpo humano son integrados en una red médica cada vez más densa. Ante esto, Foucault evalúa dos hipótesis contrapuestas. La primera hipótesis plantea que la medicina moderna es una medicina individual, desde el momento en que está penetrada por relaciones mercantiles. La medicina moderna, en la medida en que está ligada a la economía capitalista, es una medicina individualista que no conoce más que la relación mercantil médico-paciente, desconociendo la dimensión global, colectiva, social de la salud. Contra esta hipótesis, Foucault sostiene que la medicina moderna es una medicina eminentemente social, cuyo fundamento es una cierta tecnología del cuerpo social. La medicina moderna es, entonces, para Foucault, una medicina global, colectiva, social, que solo se individualiza en la relación terminal médico-paciente. En este sentido, Foucault indica:

Yo sostengo la hipótesis de que, con el capitalismo, no se ha pasado de una medicina colectiva a una medicina privada, sino que se ha producido precisamente lo contrario; el capitalismo que se desarrolla a fines del siglo dieciocho y comienzos del siglo diecinueve, ha socializado desde el inicio un primer objeto, el cuerpo, en función de la fuerza productiva, de la fuerza de trabajo. El control de la sociedad sobre los individuos no se efectúa solamente por la conciencia o por la ideología, sino también en el cuerpo y con el cuerpo. Para la sociedad capitalista, es lo bio-político lo que importaba antes que nada, lo biológico, lo somático, lo corporal. El cuerpo es una realidad bio-política; la medicina es una estrategia bio-política ${ }^{7}$.

A partir de esta hipótesis, Foucault presenta el nacimiento de la medicina social en tres momentos: primero, en el siglo dieciocho, el desarrollo de la medicina de Estado alemana, en el marco del cameralismo y la ciencia de policía; segundo, hacia fines del siglo dieciocho, el desarrollo de la higiene pública en Francia; tercero, en el siglo diecinueve, el desarrollo de la medicina laboral inglesa y su preocupación por la salud de los trabajadores ${ }^{8}$.

7 "Je soutiens l'hypothèse qu'avec le capitalisme on n'est pas passé d'une médecine collective à une médecine privée, mais que c'est précisément le contraire qui s'est produit; le capitalisme, qui se développe à la fin du XVIIIe siècle et au début du XIXe siècle, a d'abord socialisé un premier objet, le corps, en fonction de la force productive, de la force de travail. Le contrôle de la société sur les individus ne s'effectue pas seulement par la conscience ou par l'idéologie, mais aussi dans le corps et avec le corps. Pour la société capitaliste, c'est le bio-politique qui importait avant tout, le biologique, le somatique, le corporel. Le corps est une réalité bio-politique; la médecine est une stratégie bio-politique". Ibid., pp. 209-210.

${ }^{8}$ Ya en la conferencia de 1976 "Crise de la médecine ou crise de l'antimédecine?", Foucault señalaba que la medicalización moderna daba lugar a una "somatocracia" y a una "bio-historia", 
Ahora bien, en este pasaje, Foucault menciona varios de los elementos que posteriormente serán convocados cada vez que el concepto de biopolítica sea retomado. En primer lugar, la biopolítica aparece vinculada a un tratamiento no individual de los cuerpos, sino a una estrategia que opera a nivel de los procesos colectivos: el cuerpo es una realidad biopolítica desde el momento en que participa en procesos de conjunto que lo atraviesan y exceden su caso (como, por ejemplo, su integración económica en tanto fuerza de trabajo). En segundo lugar, esta noción da cuenta de un acople, una solicitación mutua entre capitalismo y medicina: la medicina se capitaliza; la sociedad capitalista se medicaliza. En tercer lugar, la biopolítica aparece como una tecnología cuya historia cubre el período que va desde la policía de Estado alemana del siglo dieciocho hasta la medicina de trabajo inglesa de fines del siglo diecinueve, desde el cameralismo alemán hasta el liberalismo decimonónico. Fenómenos globales, desarrollo capitalista, economía política: primera aparición, entonces, de la noción de biopolítica.

\section{DOS EXCURSOS BIOPOLÍTICOS: SEXUALIDAD Y GUERRA}

Dos años más tarde, la noción de biopolítica será recuperada en varias intervenciones. Entre ellas, las más salientes corresponden al primer volumen de su Histoire de la sexualité, titulado La volonté de savoir, y al curso del Collège de France de 1976, 'Il faut défendre la société'. En ambos casos, Foucault dedica dos extensos excursos a la biopolítica. Decir "excursos" es decir que la noción de biopolítica es presentada en ambos casos como un desvío del argumento principal, un paréntesis necesario a efectos de proseguir con las exposiciones centrales.

En el caso de La volonté de savoir, primer tomo de la Histoire de la sexualité, Foucault sostiene la hipótesis de que la puesta en discurso del sexo, lejos de sufrir un proceso de represión, silenciamiento o restricción, ha estado, por el contrario, sometida a un mecanismo de incitación creciente. El discurso sobre el sexo no es reprimido por el poder sino, al contrario, incitado, producido, solicitado a cada momento. Con esto, Foucault intenta reprogramar la relación sexopoder a la base de la teoría psicoanalítica y de las perspectivas freudomarxistas de su época. Foucault indica que, en la obra de Sigmund Freud (1856-1939), puede rastrearse una concepción del sexo como fondo natural, como dato sobre

indicando con ello que médicos y biólogos trabajan "al nivel de la vida misma y de sus eventos fundamentales". Michel Foucault, “Crise de la médecine ou crise de l'antimédecine?", en DefERT y EWALD (dirs.), Dits et écrits II, pp. 42, 48. 
el que opera el poder, mediante un ejercicio represivo. Foucault indica también que otras lecturas de Freud han sido posibles: Jacques Lacan (1901-1981), entre otros, ha relativizado este programa sexo-naturaleza versus poder-represión, indicando que la sexualidad se produce por el poder mismo, sin exterioridad respecto de su ley y su ejercicio represivo. De este modo, la sexualidad no es ya un dato anterior al ejercicio del poder sobre ella, sino que es en el mismo ejercicio del poder que la sexualidad se formula. De esta manera, Foucault indica que se opera un desplazamiento en lo que hace a la naturalidad del sexo pero, sin embargo, se sostiene una concepción eminentemente represiva del poder; posición que debe ser recalculada. En suma, a efectos de aprehender el dispositivo de la sexualidad, es necesario desembarazarse no solo de la naturalidad del sexo sino también del carácter represivo del poder. Es aquí donde Foucault indica el carácter productivo del poder que inviste el dispositivo de la sexualidad, descartando la hipótesis que sostiene una represión del sexo por el poder. Ahora bien, si esta hipótesis represiva no puede desmantelar el dispositivo de la sexualidad, si las luchas antirrepresivas no son más que una pericia de este mismo dispositivo, un desplazamiento interior que no lo toca en lo esencial, ¿por qué se persiste en sostener esta hipótesis represiva? ${ }^{9}$

Otras son las preocupaciones y los temas del curso 'Il faut défendre la société'. En este curso Foucault da cuenta de la emergencia del discurso histórico político en Occidente a partir de fines del siglo dieciséis. Un discurso que articula una crítica permanente al Estado, las leyes y las instituciones, identificando en ellos la prosecución de la guerra por los medios de la paz civil. Este discurso oposicional, asentado en una historia ininterrumpida de exacciones y conflictos, encuentra sus primeros portavoces en los antirrealistas del siglo diecisiete inglés, que denuncian en el poder de los Estuardo la persistencia del yugo normando impuesto sobre el pueblo sajón desde la invasión de la Casa de Hastings del siglo once ${ }^{10}$. Este discurso histórico político se manifiesta también en el siglo dieciocho francés, de la mano de la nobleza desplazada por el creciente poder monárquico; nobleza que hará uso de una historia racial de Francia, cifrando los conflictos políticos de la época en los términos de una batalla milenaria y persistente

${ }^{9}$ Michel Foucault, Histoire de la sexualité 1, pp. 172-173 [trad. esp., pp. 158-159].

${ }^{10}$ Michel Foucault, 'Il faut défendre la société'. Cours au Collège de France (1975-1976), Gallimard, Paris, 1997, pp. 75-100 [Traducción al español: Defender la sociedad. Curso en el Collège de France 1975-1976, $2^{\circ}$ reimpresión, Fondo de Cultura Económica, Buenos Aires, 2001, pp. 85-110]. 
entre francos, germanos, galos y romanos ${ }^{11}$. Ahora bien, Foucault indica que, a partir de la Revolución francesa, este discurso de función oposicional, crítica y partisana se refuncionaliza en términos de una estrategia universalizadora, centralizadora y estatal, eliminando el componente bélico del discurso histórico. De esta manera, en el relato de Foucault, el discurso histórico político deja de ser la constatación de una guerra en curso por debajo del Estado, las leyes y las instituciones; y pasa ahora a identificar en el Estado postrevolucionario la consumación de la historia en términos de la figura reconciliadora y totalizadora de la nación. Ahora bien, ¿por qué reemerge la guerra en el discurso histórico político contemporáneo ${ }^{12}$

Tenemos, de este modo, dos líneas de investigación bien diversas, con dos preguntas bien diversas también. Por un lado, la pregunta por la persistencia de la hipótesis represiva en los análisis de la sexualidad; por otro lado, la pregunta por la pervivencia de la guerra en el discurso histórico político contemporáneo. De cara a estas dos preguntas, Foucault articula una misma respuesta: es necesario considerar la emergencia del biopoder.

En ambas intervenciones, los desarrollos de Foucault son muy semejantes. Claramente, en el curso del Collège de France, Foucault estaba anticipando varios párrafos del libro que publicaría ese mismo año. De modo que ambas intervenciones pueden, sin demasiados problemas, ser aprehendidas como un mismo texto, que funciona en dos contextos bien distintos y con fines claramente diferenciado ${ }^{13}$. En ambos casos, la biopolítica se caracteriza mediante la identificación de su objeto, de los dispositivos que emplea y de su forma de normalización específica.

1. Objeto: la biopolítica consiste en una tecnología de poder que opera sobre los cuerpos pero solo en tanto participan de procesos de conjunto; una tecnología de poder vuelta a fenómenos globales como los de natalidad, fecundidad, morbilidad, vejez, accidentes, higiene pública; todos estos, fenómenos pertinentes ya no al cuerpo individual sino a la población. Mientras la mecánica soberana implica un tratamiento de las multiplicidades humanas en tanto que súbditos; y mientras las tecnologías disciplinarias operan sobre las multiplicidades en tanto que cuerpos; las tecnologías de la biopolítica aprehenden las multiplicidades en tanto que población.

${ }^{11}$ Ibid., pp. 101-148 [trad. esp., pp. 111-156].

${ }^{12}$ Ibid., p. 213 [trad. esp., p. 217].

${ }^{13}$ Ibid., pp. 213-234 [trad. esp., pp. 217-237]; FouCAULT, Histoire de la sexualité 1, pp. 177191 [trad. esp., pp. 163-176]. 
2. Dispositivos: La biopolítica opera, entonces, sobre fenómenos poblacionales, eminentemente aleatorios e imprevisibles; fenómenos que, sin embargo, son susceptibles de inscribirse en series estadísticas. Ante la observación de estas series, se disponen intervenciones a nivel de las determinaciones globales de estos fenómenos, fijando equilibrios, manteniendo promedios, estableciendo una especie de homeostasis. La regularización es, entonces, el dispositivo específico de la biopolítica, que procura estados globales de equilibrio.

3. Normalización: Dado este objeto y estos dispositivos, la forma de normalización específica a la biopolítica no es ya la de la ley soberana, que establece lo prohibido, enlazando a ello un castigo. Se trata más bien de la aplicación de una norma tanto sobre el cuerpo que se pretende disciplinar como sobre la población que se pretende regularizar. La norma articula mecanismos continuos, "reguladores y correctivos"14. Se trata de una norma que, por un lado corrige a los cuerpos y, por otro lado, regulariza a las poblaciones: "la sociedad de normalización es una sociedad donde se cruzan, según una articulación ortogonal, la norma de la disciplina y la norma de la regulación"15.

Hecha esta caracterización de la biopolítica, Foucault recupera las preguntas que dieron lugar a su tratamiento. En el caso de La volonté de savoir, Foucault señala que la sexualidad aparece como un dispositivo bisagra, un dispositivo que responde y opera tanto en términos disciplinarios como en términos biopolíticos. El dispositivo de la sexualidad es un dispositivo atento a cada cuerpo: vigilancia, sanción, examen se ciernen sobre cada caso, operando un disciplinamiento corporal insidioso, permanente, atento al detalle. A su vez, la sexualidad también concierne a fenómenos globales de fecundidad, natalidad, morbilidad, higiene. De este modo, el sexo aparece como el fondo de un juego político, atento a la vida del individuo y de la población. La sexualidad, entonces, no es lo reprimido por el poder sino, al contrario, su dispositivo dilecto, el punto de convergencia entre el disciplinamiento de cada cuerpo y la regularización de las poblaciones.

De manera convergente, en una entrevista publicada en enero de 1977, en ocasión de la publicación de La volonté de savoir, Foucault indica que la preo-

${ }^{14}$ Foucault, Histoire de la sexualité 1, p. 189 [trad. esp., p. 174].

15 "La société de normalisation, c'est une société où se croisent, selon une articulation orthogonale, la norme de la discipline et la norme de la régulation". FouCAULT, 'Il faut défendre la société', p. 225 [trad. esp., p. 229]. Deliberadamente austeros en este punto. Es que las intervenciones de 1976 no brindan mayores precisiones sobre el tipo de normalización que corresponde a las disciplinas y a la biopolítica. La diferencia será establecida posteriormente en el curso de 1978. FouCAULT, Sécurité, territoire, population, p. 59 [trad. esp., p. 76]. 
cupación esencial de su libro es la de dar cuenta de un poder que se ejerce sobre el cuerpo mismo:

Lo que yo busco es intentar mostrar cómo las relaciones de poder pueden pasar materialmente por el espesor mismo de los cuerpos sin tener que ser transmitidas por la representación de los sujetos. Si el poder alcanza a los cuerpos, no es porque haya estado interiorizado desde el principio en la conciencia de la gente. Hay una red de bio-poder, de somato-poder que es ella misma una red a partir de la cual nace la sexualidad como fenómeno histórico y cultural al interior del cual nos reconocemos y nos perdemos ${ }^{16}$.

De este modo, el dispositivo de la sexualidad enlaza los poderes disciplinario y biopolítico sobre la vida de cuerpos y poblaciones. La relación que el poder entabla con el sexo no es la de una represión o la de un llamado a la censura. Es la de una producción e incitación constantes. De este modo, la hipótesis represiva no hace más que reproducir la lógica disciplinaria y biopolítica del dispositivo de la sexualidad. "Ironía del dispositivo: nos hace creer que en ello reside nuestra "liberación""17. Esto, respecto de Histoire de la sexualité.

En el caso de 'Il faut défendre la société', el excurso sobre la biopolítica permite a Foucault indicar la condición de existencia de la guerra al interior del discurso histórico-político de la nación, concebida en los términos de consumación totalizadora. La profunda mutación del poder contemporáneo implicada en la emergencia de las disciplinas y, posteriormente, de la biopolítica configuran un ejercicio productivo del poder, un ejercicio del poder que no se asienta en la represión sino en el fortalecimiento de la vida de los cuerpos y poblaciones que transige; en suma, un ejercicio del poder consistente no en "hacer morir y dejar vivir" sino en "hacer vivir y dejar morir". En este contexto, ¿cuáles son las condiciones de aceptabilidad del ejercicio del poder de muerte? Foucault indica que

16 "Ce que je cherche, c'est à essayer de montrer comment les rapports de pouvoir peuvent passer matériellement dans l'épaisseur même des corps sans avoir à être relayés par la représentation des sujets. Si le pouvoir atteint le corps, ce n'est pas parce qu'il a d'abord été intériorisé dans la conscience des gens. Il y a un réseau de bio-pouvoir, de somato-pouvoir qui est lui-même un réseau à partir duquel naît la sexualité comme phénomène historique et culturel à l'intérieur duquel à la fois nous nous reconnaissons et nous nous perdons". Michel FouCAUlt, "Les rapports de pouvoir passent à l'intérieur des corps", en DEFERT y EWALD (dirs.), Dits et écrits II, p. 231 [Traducción al español: Michel foucault, "Las relaciones de poder penetran en los cuerpos", en Microfísica del poder, $3^{\circ}$ edición, La Piqueta, Madrid, 1992, p. 166].

17 "Ironie de ce dispositif: il nous fait croire qu'il y va de notre 'libération"'. FouCAULt, Historie de la sexualité 1, p. 211 [trad. esp., p. 194]. 
el "hacer morir" sólo es aceptable cuando se presenta como vital, como subsidiario de un poder que se ejerce de manera preeminente mediante el "hacer vivir". Es en este contexto que el discurso histórico político contemporáneo reactiva la guerra de razas. El racismo aparece así como el fondo del juego político, como el intercambiador entre poder de muerte y poder sobre la vida: se mata a unos para fortalecer la vida de otros. Convergentemente, en un seminario dictado en la Universidad de Vermont en 1982, Foucault indica que el Estado:

[E]jerce su poder sobre los seres vivientes en tanto que seres vivientes, y su política es, necesariamente, una biopolítica. La población, que no es más que aquello sobre lo que vela el Estado en su propio interés, bien entendido, puede ser masacrada por el mismo Estado, de ser necesario. La tanatopolítica es, de esta manera, el reverso de la biopolítica ${ }^{18}$.

Es entonces en defensa de la vida de la nación que algunos deben morir. Solo de esta manera es comprensible el retorno de la guerra al interior de un discurso histórico que postula la unidad nacional.

En suma, el episodio biopolítico funciona en estas intervenciones a efectos de aprehender la contemporaneidad de las investigaciones en curso. Ya se trate del dispositivo de la sexualidad o del discurso histórico político, la biopolítica opera en ambos casos como un excurso, un incidente que debe ser recuperado a efectos de iluminar otra $\operatorname{cosa}^{19}$. Incidencia determinante de la biopolítica sobre el dispositivo de la sexualidad, sobre el historicismo político. Carácter incidental, también, de la biopolítica respecto de estas investigaciones ${ }^{20}$.

18 'Il [l'État] exerce son pouvoir sur les êtres vivants en tant qu'êtres vivants, et sa politique est, en conséquence, nécessairement une biopolitique. La population n'étant jamais que ce sur quoi veille l'État dans son propre intérêt, bien entendu, l'État peut, au besoin, la massacrer. La thanatopolitique est ainsi l'envers de la biopolitique". Michel FouCAult, "La technologie politique des individus", en DeFERT y EwALD (dirs.) Dits et écrits II, p. 1645.

${ }^{19}$ Tanto es así, que en Defender la sociedad Foucault pide disculpas por haberse extendido tanto en el tratamiento de la biopolítica. Ver Foucault, 'Il faut défendre la société', p. 226 [trad. esp., p. 229].

${ }^{20}$ Una objeción posible en este punto: ante la pregunta de Alain Grosrichard respecto de la relevancia del último capítulo de Histoire de la sexualité 1, Foucault responde: "Sí, esta última parte, nadie habla de ella. Sin embargo, el libro es corto, pero sospecho que mucha gente no ha lle gado nunca a ese capítulo. Y es, a pesar de todo, el fondo del libro". ["Oui, cette dernière partie, personne n'en parle. Pourtant, le livre est court, mais je soupçonne les gens de n'être jamais arrivés jusqu'à ce chapitre. C'est tout de même le fond du livre"]. Michel Foucault, "Le jeu de Michel Foucault", en DEFERT y EWALD (dirs.), Dits et écrits II, p. 323. Cualquiera sea el sentido de la palabra fondo en este contexto, es de notarse que, de allí en más, entrevistadores y entrevistado se inte 
En octubre de ese mismo año, Foucault publica en el periódico Le Monde una reseña del libro De la biologie a la culture del médico y antropólogo Jacques Ruffié $^{21}$. En esta pequeña y elogiosa reseña, Foucault recupera dos conceptos centrales del libro de Ruffié. Se trata de los conceptos de especie y de raza. En su lectura del libro de Ruffié, Foucault indica que la especie humana aparece ante la biología como un todo poliforme, inestable; como un conjunto de variaciones constantes, de comunicaciones, enlaces, mezclas al interior de un patrimonio genético común. En este contexto, la evidencia biológica recabada por el libro indica que las razas nunca han existido en la especie humana: en la naturaleza no hay razas. El racismo, en este sentido, no es un discurso que se apoya sobre el dato natural, biológico; sino que es un discurso político, que establece una cesura irremediablemente arbitraria sobre el continuo de la especie humana. Es en esta línea que Foucault recupera el concepto de biopolítica:

La humanidad es un 'pool de genes intercomunicantes': de poblaciones, es decir, de conjuntos de variaciones que no cesan de formarse y de deshacerse. Es la historia la que dibuja estos conjuntos antes de borrarlos; no hay que buscar unos hechos biológicos brutos y definitivos que, desde el fondo de la 'naturaleza', se impondrían a la historia. La obra de Jacques Ruffié contiene otros análisis de este tipo. Todos son importantes: dado que allí se ven formuladas con toda claridad las cuestiones de una 'bio-historia' que no sería más la historia unitaria y mitológica de la especie humana a través del tiempo y una 'bio-política' que no sería aquélla de las particiones, las conservaciones y las jerarquías, sino aquella de la comunicación y de los polimorfismos ${ }^{22}$.

En esta pequeña reseña, Foucault plantea la posibilidad de otra biopolítica, distinta de la del racismo de Estado. De esta manera, identifica nuevamente a la

\footnotetext{
resarán exclusivamente en el problema del racismo, en el pasaje de la simbólica de la sangre a la analítica de la sexualidad; la noción de biopolítica no es siquiera mencionada.

${ }^{21}$ Michel Foucault, "Bio-historie et bio-politique", en Defert y Ewald (dirs.), Dits et écrits II, pp. 95-97.

22 "L'humanité est un «pool de gènes intercommunicants»: des populations, c'est-à-dire des ensembles de variations, ne cessent de s'y former et de s'y défaire. C'est l'histoire qui dessine ces ensembles avant de les effacer; il ne faut pas y chercher des faits biologiques bruts et définitifs qui, du fond de la «nature», s'imposeraient à l'histoire. L'ouvrage de Jacques Ruffié contient bien d'autres analyses de ce genre. Toutes sont importantes; car on y voit s'y formuler en toute clarté les questions d'une «bio-histoire» qui ne serait plus l'histoire unitaire et mythologique de l'espèce humaine à travers le temps et une «bio-politique» qui ne serait pas celle des partages, des conservations et des hiérarchies, mais celle de la communication et du polymorphisme". Ibid., p. 97.
} 
biopolítica como un poder que actúa sobre las poblaciones, en tanto "conjuntos de variaciones". Un poder sobre la vida que, en el caso del racismo, opera mediante particiones, conservaciones y jerarquías. Contra la biopolítica racial, el libro de Ruffié reseñado por Foucault indicaría la posibilidad de otra política sobre la vida, atenta a la comunicación y los polimorfismos que caracterizan a la especie humana. En sus investigaciones posteriores, Foucault no volverá sobre esta idea de otra política sobre la vida.

\section{BIOPOLÍTICA Y ECONOMÍA}

Ahora bien, en estas intervenciones, Foucault insiste en la correlación entre nacimiento de la biopolítica y desarrollo capitalista. En el primer tomo de Histoire de la sexualité se señala al poder de hacer vivir y dejar morir como un elemento indispensable en el desarrollo del capitalismo. Disciplinas y biopolítica contribuyen ambas a la acumulación de cuerpos necesaria a efectos de la acumulación del capital; evidenciando un ajuste, una solicitación mutua entre biopoder y desarrollo capitalista. En el curso 'Il faut défendre la société', Foucault recupera también esta imbricación. Recupero una extensa cita:

[T]odo sucedió como si el poder, que tenía la soberanía como modalidad y esquema organizativo, se hubiera demostrado inoperante para regir el cuerpo económico y político de una sociedad en vías de explosión demográfica e industrialización a la vez. De manera que muchas cosas escapaban a la vieja mecánica del poder de soberanía, tanto por arriba como por abajo, en el nivel del detalle y en el de la masa. Para recuperar el detalle se produjo una primera adaptación: adaptación de los mecanismos de poder al cuerpo individual, con vigilancia y adiestramiento; eso fue la disciplina. Se trató, desde luego, de la adaptación más fácil, la más cómoda de realizar. Por eso fue la más temprana —en el siglo diecisiete y principios del dieciocho- en un nivel local, en formas intuitivas, empíricas, fraccionadas, y en el marco limitado de instituciones como la escuela, el hospital, el cuartel, el taller, etcétera. Y a continuación, a fines del siglo dieciocho, tenemos una segunda adaptación, a los fenómenos globales, los fenómenos de población, con los procesos biológicos o biosociológicos de las masas humanas. Adaptación mucho más difícil porque implicaba, desde luego, órganos complejos de coordinación y centralización ${ }^{23}$.

23 “"[T]out s'est passé comme si le pouvoir, qui avait comme modalité, comme schéma organisateur, la souveraineté, s'était trouvé inopérant pour régir le corps économique et politique d'une 
De modo que las disciplinas y la biopolítica aparecen como tecnologías de poder adecuadas al desarrollo capitalista. Con esto Foucault no indica el proyecto o la estrategia de una clase (notar el "todo sucedió como si..."); tampoco establece una determinación económica de las tecnologías de poder. Más bien, sugiere que fueron estas tecnologías de poder las que hicieron posible el desarrollo capitalista; capitalismo que a su vez catalizó, reforzó, profundizó el desarrollo del biopoder. En todo caso, es posible indicar que capitalismo y biopoder emergen ambos de unas mismas condiciones de existencia: las expansiones demográficas y las crisis económicas que irrumpen durante la época clásica. Ambos términos aparecen así subtendidos en la simultaneidad de unas condiciones de existencia comunes y en la solidaridad cruzada de sus determinaciones.

Como sea el caso, en ese mismo año, Foucault dicta una conferencia en la Universidad de Bahía donde retoma el concepto de biopolítica también en relación al capitalismo ${ }^{24}$. Se indica en este seminario la obsolescencia técnica del poder de soberanía de cara al capitalismo emergente; un poder de soberanía discontinuo y oneroso, que deja escapar el detalle y los procesos globales. Ante esto, nuevamente se identifica la emergencia de las disciplinas y, posteriormente, de la biopolítica.

Intento mostrar...cómo esta mutación de la tecnología del poder forma parte absolutamente del desarrollo del capitalismo. Ella forma parte de este desarrollo en la medida que, por un lado, es el desarrollo del capitalismo lo que ha hecho necesaria esta mutación tecnológica, pero esta mutación ha hecho posible el desarrollo

\footnotetext{
société en voie, à la fois, d'explosion démographique et d'industrialisation. Si bien qu'à la vieille mécanique du pouvoir de souveraineté beaucoup trop de choses échappaient, à la fois par en bas et par en haut, au niveau du détail et au niveau de la masse. C'est pour rattraper le détail qu'une première accommodation a eu lieu: accommodation des mécanismes de pouvoir sur le corps individuel, avec surveillance et dressage -cela a été la discipline. Bien sûr, cela a été l'accommodation la plus facile, la plus commode à réaliser. C'est pourquoi elle s'est réalisée le plus tôt - dès le XVIIe, début du XVIIIe siècle - à un niveau local, dans des formes intuitives, empiriques, fractionnées, et dans le cadre limité d'institutions comme l'école, l'hôpital, la caserne, l'atelier, etc. Et puis vous avez ensuite, à la fin du XVIIIe siècle, une seconde accommodation, sur les phénomènes globaux, sur les phénomènes de population, avec les processus biologiques ou bio-sociologiques des masses humaines. Accommodation beaucoup plus difficile car, bien entendu, elle impliquait des organes complexes de coordination et de centralisation". Foucault, 'Il faut défendre la société', pp. 222-223 [trad. esp., p. 226].

${ }^{24}$ Michel Foucault, "Les mailles du pouvoir", en Defert y Ewald (dirs.) Dits et écrits II, pp. 1001-1020 [Traducción al español: "Las mallas del poder", en Michel FoucAuLt, Obras esenciales III: Estética, ética, hermenéutica, Paidós, Barcelona, 1999, pp. 235-254].
} 
del capitalismo; en suma, una implicación permanente de dos movimientos, que son de una cierta manera adaptados el uno al otro ${ }^{25}$.

A partir de estas intervenciones, vemos reiterarse las características de la biopolítica: una tecnología de poder que se da por objeto a las poblaciones; por dispositivo a la regularización; un poder que normaliza cuerpos y poblaciones; un poder imbricado, enlazado íntimamente con el desarrollo capitalista.

Ahora bien, en los cursos del Collège de France de 1978 y 1979, Foucault se propone trabajar las nociones de biopoder y biopolítica. No estaríamos ya ante un tratamiento episódico, incidental, sino ante un par de cursos que tienen por objeto a la biopolítica. Se trata de los cursos Sécurité, territoire, population (1978) y Nacimiento de la biopolítica (1979). En ambos, Foucault se ve en la necesidad de abordar de frente esta mutación tecnológica fundamental implicada por el ingreso de la vida en los mecanismos y cálculos del poder. En la primera clase de Sécurité, territoire, population, Foucault explicita:

Este año querría comenzar el estudio de algo que hace un tiempo llamé, un poco en el aire, biopoder, es decir una serie de fenómenos que me parece bastante importante, a saber, el conjunto de mecanismos por medio de los cuales aquello que, en la especie humana, constituye sus rasgos biológicos fundamentales, podrá ser parte de una política, una estrategia política, una estrategia general de poder; en otras palabras, cómo, a partir del siglo XVIII, la sociedad, las sociedades occidentales modernas, tomaron en cuenta el hecho biológico fundamental de que el hombre constituye una especie humana. Esto es, en líneas generales, lo que llamo, lo que he llamado biopoder ${ }^{26}$.

25 “J'ai essayé de montrer le contraire, c'est-à-dire comment cette mutation de la technologie du pouvoir fait absolument partie du développement du capitalisme. Elle fait partie de ce développement dans la mesure où, d'un côté, c'est le développement du capitalisme qui a rendu nécessaire cette mutation technologique, mais cette mutation a rendu possible le développement du capitalisme, bref, une implication permanente des deux mouvements, qui sont d'une certaine façon engrenés l'un dans l'autre". Ibid., p. 1019 [trad. esp., pp. 252-253].

26 "Cette année, je voudrais commencer l'étude de quelque chose que j'avais appelé comme ça, un petit peu en l'air, le bio-pouvoir, c'est-à-dire, cette série de phénomènes qui me paraît assez importante, à savoir l'ensemble des mécanismes par lesquels ce qui, dans l'espèce humaine, constitue ses traits biologiques fondamentaux va pouvoir entrer à l'intérieur d'une politique, d'une stratégie politique, d'une stratégie générale de pouvoir, autrement dit, comment la société, les sociétés occidentales modernes, à partir du XVIIIe siècle, ont repris en compte le fait biologique fondamental que l'être humain constitue une espèce humaine. C'est en gros ça que j'appelle, que j'ai appelé, comme ça, le bio-pouvoir”. Foucault, Sécurité, territoire, population, p. 3 [trad. esp., pp. 15-16]. 
A efectos del estudio del biopoder, Foucault dedica tres clases al análisis de los dispositivos de seguridad, refinamiento y profundización de los dispositivos regularizadores identificados con el ejercicio de la biopolítica. Pero, a partir de la cuarta clase del curso, Foucault se dedica al trabajo de las nociones de gobierno y gubernamentalidad, que comprometen el resto de la cursada. De este modo, el estudio del biopoder queda desplazado en función de una historia de la gubernamentalidad. En su curso del año siguiente, Nacimiento de la biopolítica, Foucault se propone retomar la historia de la gubernamentalidad liberal a efectos de un estudio de la biopolítica. Pero ya en la primera sesión anticipa: "creí que este año podía hacer un curso sobre la biopolítica;" ${ }^{27} \mathrm{y}$, tras dos meses de dictado de clases, admite: "les aseguro que en un comienzo tuve en verdad la intención de hablarles de biopolítica pero después como las cosas son lo que son, resulta que terminé por hablarles extensamente -demasiado extensamente tal vez- del neoliberalismo" 28 . De esta manera, Foucault atraviesa ambos cursos sin elaborar una genealogía de la biopolítica. En ambos casos, la operación es la misma. De cara al estudio de la biopolítica, se hace necesario comenzar reponiendo la historia de la gubernamentalidad en la que ésta se inscribe; una historia que exige un tratamiento extenso, prorrogado, creciente; y que finalmente no deja lugar para el estudio de la biopolítica en sí. Todo sucede como si la hipótesis biopolítica, para ser efectiva, reclamara, exigiera su inscripción en un marco más amplio ${ }^{29}$; este marco está constituido por la gubernamentalidad, entendida como el conjunto de procedimientos, saberes y tácticas que tienen por objeto a la población, por dispositivo a la seguridad y por forma de saber a la economía política ${ }^{30}$. Seguridad, economía, población constituyen las coordenadas del poder bajo la forma del gobierno. Es en la inserción en este marco más amplio que la biopolítica puede ser estudiada.

Ahora bien, ¿en qué consisten los dispositivos de seguridad? Foucault dedica las primeras tres clases de 1978 a trabajar en torno a estos dispositivos, identificando su tratamiento del espacio, del tiempo, de la multiplicidad y de la norma $^{31}$, en contraste permanente con la mecánica soberana y la tecnología disciplinaria.

27 "J'avais pensé pouvoir vous faire cette année un cours sur la biopolitique". FouCAULT, Naissance de la biopolitique, p. 23 [trad. esp., p. 40].

28 'Je voudrais vous assurer, malgré tout, que j'avais bien l'intention, au départ, de vous parler de biopolitque et puis, les choses étant ce qu'elles sont, voilà que j'en suis arrivé à vous parler longuement, et trop longuement peut-être, du néolibéralisme”. Ibid., p. 191 [trad. esp., p. 217].

${ }^{29}$ Esto es indicado en Michel Senellart, "Situation des cours", en Foucault, Sécurité, territoire, population, pp. 381-410 [trad. esp., pp. 417-453].

${ }^{30}$ FoucAult, Sécurité, territoire, population, pp. 111-112 [trad. esp., p. 136].

${ }^{31}$ Ibid., p. 13 [trad. esp. 27]. 
En primer lugar, entonces, los espacios de seguridad. Los dispositivos de seguridad operan sobre el territorio a partir de su acondicionamiento, disponiendo una serie de intervenciones destinadas a estimular y catalizar ciertas circulaciones mientras se dificultan y se desfavorecen ciertas otras. Tomando el ejemplo de los proyectos de urbanismo del siglo dieciocho, Foucault indica que estos dispositivos trabajan observando las regularidades en la circulación de personas $\mathrm{y}$ cosas en el territorio, identificando los amontonamientos, las dispersiones; y disponiendo intervenciones sobre el medio que favorecen la higiene pública, el intercambio comercial, la vigilancia. Si el poder de soberanía opera sobre el territorio fijando límites y jerarquías; si, por otro lado, la tecnología disciplinaria opera sobre un territorio saturado de reticulaciones que permiten una vigilancia constante; los dispositivos de seguridad intervienen acondicionando el medio.

Los dispositivos de seguridad trabajan, fabrican, organizan, acondicionan un medio aun antes de que la noción se haya constituido y aislado. El medio será entonces el ámbito en el cual se da la circulación. Es un conjunto de datos naturales, ríos, pantanos, colinas, y un conjunto de datos artificiales, aglomeración de individuos, aglomeración de casas, etc. El medio es una cantidad de efectos masivos que afectan a quienes residen en él... El medio aparece por último como un campo de intervención donde, en vez de afectar a los individuos como un conjunto de sujetos de derecho capaces de acciones voluntarias -así sucedía con la soberanía-, en vez de afectarlos como una multiplicidad de organismos, de cuerpos susceptibles de prestaciones, y de prestaciones exigidas como en la disciplina, se tratará de afectar, precisamente, a una población ${ }^{32}$.

Segunda coordenada de los dispositivos de seguridad: el tiempo, el tratamiento del acontecimiento, de lo aleatorio. En el caso de las disciplinas, se articula un tiempo saturado, de vigilancias e intervenciones permanentes en función de una captura total del tiempo y de una determinación minuciosa de fases evo-

32 "Les dispositifs de sécurité travaillent, fabriquent, organisent, aménagent un milieu avant même que la notion ait été formée et isolée. Le milieu, ça va être donc ce en quoi se fait la circulation. Le milieu, c'est un ensemble de données naturelles, fleuves, marécages, collines, c'est un ensemble de données artificielles, agglomération d'individus, agglomération de maisons, etc. Le milieu, c'est un certain nombre d'effets qui sont des effets de masse portant sur tout ceux qui y résident...le milieu apparaît comme un champ d'intervention où, au lieu d'atteindre les individus comme un ensemble de sujets de droit capables d'actions volontaires -ce qui était le cas de la souveraineté-, au lieu de les atteindre comme une multiplicité d'organismes, de corps susceptibles de performances, et de performances requises comme dans la discipline, on va essayer d'atteindre, précisément, une population". Ibid., pp. 22-23 [trad. esp., pp. 40-41]. 
lutivas, desarrollos esperables, intervenciones correctivas. El tratamiento del tiempo por los dispositivos de seguridad es bien distinto al de las disciplinas: en este caso, se opera mediante la disposición de series abiertas, donde es posible observar acontecimientos, fenómenos aleatorios, probabilidades, ocurrencias incalculadas. Lo aleatorio no es expulsado del tiempo de seguridad sino que, más bien, es incorporado en sus probabilidades. No se trata, entonces, de una captura saturada del tiempo en función de un programa disciplinario sino de una observación de los fenómenos regulares y aleatorios que permite un recálculo constante de series abiertas y de las disposiciones sobre el medio a efectos de favorecer ciertos fenómenos en detrimento de otros.

En tercer lugar, los dispositivos de seguridad implican un tratamiento de la multiplicidad no en tanto súbditos, tampoco en tanto cuerpos, sino en tanto población. Es la población el objeto de estos dispositivos, el nivel pertinente para el desarrollo de su análisis y ejercicio. Se establece así una cesura entre los niveles de intervención. La seguridad es indiferente a los fenómenos individuales; a ella atañen los procesos globales, los procesos poblacionales que permiten calcular probabilidades, recurrencias, costos de intervención. Ya no se trata de curar al enfermo, educar al estudiante, castigar al delincuente. Se trata, más bien, de controlar las endemias, mantener tasas de escolarización adecuadas, reducir las tasas de criminalidad a umbrales aceptables. No es el súbdito ni el cuerpo sino la población el objeto y el nivel de intervención pertinente de los dispositivos de seguridad.

Por último, los dispositivos de seguridad articulan una forma de normalización específica. No se trata ya de establecer de antemano lo prohibido o lo anormal y, en función de este programa, castigar o normalizar. Se trata de partir de la observación de las series estadísticas, de las probabilidades, de las distribuciones normales y, en función de ellas, intervenir sobre los casos desviados. No se trata de imponer la norma a la realidad sino de solicitarle a la realidad la definición de lo normal, a efectos de la intervención sobre el medio, lo aleatorio, la población.

En suma, los dispositivos de seguridad vienen a refinar, a ajustar el análisis de los dispositivos de regularización indicados por Foucault en sus intervenciones anteriores; y permiten, de esta manera, ajustar las características de la tecnología biopolítica en función de los siguientes elementos:

1. Objeto: se trata, al igual que en sus intervenciones anteriores, de la población, de sus fenómenos de conjunto. Es la población el objeto y el nivel de intervención pertinente de la tecnología biopolítica. 
2. Dispositivos: el refinamiento de los dispositivos de regularización en términos de dispositivos de seguridad brinda ahora una serie de precisiones respecto del tratamiento del espacio, del tiempo, de la multiplicidad y de la norma: operaciones sobre el medio, tratamiento de lo aleatorio, cifra de la multiplicidad en términos de población y normalización aparecen como las coordenadas de los dispositivos de seguridad que articula la biopolítica.

3. Normalización: Ya no se trata de la "normación" 33 disciplinaria, que impone a la realidad un modelo definido de antemano donde se distingue normal y anormal, sino que se trata de una "normalización" de seguridad, que desplaza y recalcula constantemente sus coordenadas en función de regularidades estadísticamente observables. Así, mientras las disciplinas parten de una determinación prescriptiva de lo normal que aplican en segundo lugar sobre los cuerpos, la biopolítica parte de la observación estadística de las distribuciones normales y, en función de ellas, modula su intervención.

A estas precisiones respecto del objeto, los dispositivos y la normalización biopolítica, Foucault agrega una caracterización ulterior, vinculada al marco de racionalidad al interior del cual la biopolítica es posible. Este marco de racionalidad es identificado con el término de gubernamentalidad, definido como:

[E]l conjunto constituido por las instituciones, los procedimientos, análisis y reflexiones, los cálculos y las tácticas que permiten ejercer esa forma bien específica, aunque muy compleja, de poder que tiene por blanco principal la población, por forma mayor de saber la economía política y por instrumento técnico esencial los dispositivos de seguridad ${ }^{34}$.

33 “'Autrement dit, il y a un caractère primitivement prescriptif de la norme et c'est par rapport à cette norme posée que la détermination et el repérage du normal et de l'anormal deviennent possibles. Ce caractère premier de la norme par rapport au normal, le fait que la normalisation disciplinaire aille de la norma au partage final du normal et de l'anormal, c'est à cause de cela que j'aimerais mieux dire, à propos de ce qui se passe dans les techniques disciplinaires, qu'il s'agit d'une normation plus que d'un normalisation". Ibid., p. 59 [trad. esp., p. 76: "En otras palabras, lo primero y fundamental en la normalización disciplinaria no es lo normal y lo anormal, sino la norma. Para decirlo de otra manera, la norma tiene un carácter primariamente prescriptivo, y la determinación y el señalamiento de lo normal y lo anormal resultan posibles con respecto a esa norma postulada. A causa de ese carácter primario de la norma en relación con lo normal, el hecho de que la normalización disciplinaria vaya de la norma a la diferenciación final de lo normal y lo anormal, me gustaría decir, acerca de lo que ocurre en las técnicas disciplinarias, que se trata más de una normación que de una normalización”].

34 "Par 'gouvernementalité', j'entends l'ensemble constitué par les institutions, les procédures, analyses et réflexions, les calculs et les tactiques qui permettent d'exercer cette forme bien spé- 
La noción de gubernamentalidad implica un cambio de perspectiva en los análisis de Foucault. A partir de su ocurrencia, Foucault dejará de preocuparse centralmente por los mecanismos y tecnologías de poder, para concentrarse en las racionalidades específicas que subtienden y articulan su ejercicio. De este modo, la pregunta ¿cómo se ejerce el poder? —y su respuesta por vía del análisis de los mecanismos y tecnologías disciplinarios y biopolíticos- se verá desplazada por la pregunta ¿cómo se racionaliza el ejercicio del poder? - y su respuesta por vía de una historia de las artes de gobierno ${ }^{35}$. Esta historia de las artes de gobierno, esta historia de la gubernamentalidad, abrirá un novedoso campo de investigaciones, que se remontará al análisis del poder pastoral en Occidente, caracterizado como un poder ejercido sobre un rebaño en vistas a su propia conservación y salvación. En el poder pastoral, Foucault asienta su genealogía de un arte de gobierno de las multiplicidades humanas, que encuentra continuidad en las doctrinas de la razón de Estado, de la policía de Estado, del mercantilismo y del liberalismo y neoliberalismo ${ }^{36}$. Esta genealogía de las artes de gobierno, que es al mismo tiempo una historia de la economía política, le permite a Foucault identificar una racionalidad gubernamental que conserva los rasgos del pastorado; esto es, los rasgos de un poder ejercido sobre una multiplicidad de seres vivientes, en vistas a la conservación de sus vidas y, por esta vía, a su salvación. Queda claro en qué sentido la economía política puede constituir el marco de racionalidad del ejercicio del biopoder. Como sea el caso, el interés de Foucault por trazar esta historia de la gubernamentalidad explica el relegamiento de sus investigaciones sobre la biopolítica.

\section{LA INCIDENCIA DE LA BIOPOLÍTICA}

En suma, la obra publicada de Michel Foucault permite identificar los rasgos de esta novedosa mutación en el poder en Occidente, vinculada a la emergencia de

cifique, quoique très complexe, de pouvoir qui a pour cible la population, pour forme majeure de savoir l'économie politique, pour instrument technique essentiel les dispositifs de sécurité". FouCAULT, Sécurité, territoire, population, p. 111 [trad. esp., p. 136].

${ }^{35}$ Michel Foucault, “'Omnes et singulatim': towards a criticism of political reason”, en DEFERT y EWALD (dirs.) Dits et écrits II, pp. 953-980 [Traducción al español: ““Omnes et singulatim': hacia una crítica de la razón política”, en ¿Qué es la ilustración?, La piqueta, Madrid, 1996, pp. 17-66].

${ }^{36}$ Foucault, Securité, territoire, population, passim; FouCAULT, Naissance de la biopolitique, passim. 
la biopolítica. Permite identificar su objeto, sus dispositivos, su forma de normalización específica, su marco de racionalidad. Ahora bien, no hay en la obra publicada de Foucault una genealogía de la biopolítica; no hay en su obra una analítica sistemática, extendida, rigurosa del biopoder. Es el mismo Foucault quien indica esta ausencia. En una entrevista de 1983, Foucault explicita que sus investigaciones están determinadas por una elección ético política central, que es la de determinar a cada paso cuáles son los principales peligros. Ante esta definición, los entrevistadores le preguntan si no sería lógico dar lugar a una genealogía del biopoder. Foucault responde: "No tengo tiempo para hacer eso ahora, pero podría hacerse. De hecho, debo hacerlo" ${ }^{37}$. No deja de ser sugerente que, cuando Foucault revise la traducción francesa de esta entrevista, opte por eliminar toda referencia a la biopolítica ${ }^{38}$.

Hemos, hasta aquí, sostenido la hipótesis de una biopolítica incidental. Esto no obsta, sin embargo, a la plausibilidad de sostener la incidencia de la biopolítica. Es que cada vez que Foucault identifica nuevos temas, problemas o conceptos, lo hace poniendo en marcha una relectura de su obra anterior, recuperando una serie de elementos y redistribuyéndolos en dirección a estas nuevas preocupaciones. En este sentido, es posible decir que gran parte de los elementos constitutivos de la biopolítica se encuentran presentes desde un principio en la obra de Foucault. Si la biopolítica es algo más que un incidente menor al interior de su obra, si puede ser un tema de amplia incidencia, es precisamente porque en ella se recupera y se reordena una serie de elementos persistentes todo a lo largo de las investigaciones foucauldianas. A título meramente indicativo y sin ánimos de proseguir esta línea de investigaciones, es posible conmutar los antecedentes de la biopolítica al interior de sus cuatro elementos característicos.

1. Objeto. La población constituye una preocupación constante en la obra de Foucault. Esta noción ya es problematizada en su tesis doctoral, Histoire de la folie à l'âge classique, allí donde Foucault relata el proceso de diferenciación de la locu-

37 "I have no time for that now, but it could be done. In fact, I have to do it". Michel FouCAULT, "On the genealogy of ethics. An overview of work in progress", en Hubert DREYFUS y Paul RABINOw, Michel Foucault. Beyond Structuralism and Hermeneutics, $2^{\circ}$ edición, Chicago University Press, Chicago, 1983, p. 232 [Traducción al español: Michel Foucault, "Sobre la genealogía de la ética", en Hubert Dreyfus y Paul Rabinow, Michel Foucault. Más allá del estructuralismo y la hermenéutica, Nueva Visión, Buenos Aires, 2003, p. 264].

${ }^{38}$ Michel Foucault, “À propos de la généalogie de l'éthique: un aperçu du travail en cours", en Defert y Ewald (dirs.) Dits et écrits II, p. 1431. 
ra respecto de otras formas de la sinrazón ${ }^{39}$. El problema de las poblaciones reaparece en Naissance de la clinique, cuando Foucault identifica la puesta en tela de juicio de los hospitales ${ }^{40}$. En ambos casos, Foucault ciñe un mismo período (fines del siglo dieciocho) y recupera un mismo debate (el debate económico en torno a las instituciones de asistencia). La noción de población aparece lateralmente en Les mots et les choses, en el momento en que Foucault considera la emergencia de la economía política ${ }^{41}$. Finalmente, todo su análisis respecto del poder disciplinario desplegado en Surveiller et punir está subtendido por la expansión demográfica del siglo dieciocho y la necesidad de fijar la masa de población flotante al aparato productivo ${ }^{42}$.

2. Dispositivos. Como es explícito en su conceptualización de la biopolítica, el gran antecedente de esta tecnología viene dado por la medicina ${ }^{43}$. De modo que los dispositivos de regulación y de seguridad han de rastrearse en todos los pasajes en los que Foucault trata la medicalización de Occidente. Habrá de considerarse Naissance de la clinique, especialmente aquellos pasajes referidos a la emergencia de la probabilística ${ }^{44}$. En el curso dictado en el Collège de France en 1975, Foucault describe la medicalización del poder judicial y de la familia ${ }^{45}$. Por último,

${ }^{39}$ Michel Foucault, Histoire de la folie à l'âge classique, Gallimard, Paris, 1972, pp. 477524 [Traducción al español: Historia de la locura en la época clásica, 2 tomos, $5^{\circ}$ reimpresión, Fondo de Cultura Económica, Buenos Aires, 2004, tomo 2, pp. 66-123].

${ }^{40}$ Michel Foucault, Naissance de la clinique. Une archéologie du regard médical, PUF, Paris, 1963, pp. 37-52 [Traducción al español: El nacimiento de la clínica. Una arqueología de la mirada médica, $1^{\circ}$ reimpresión, Siglo XXI, Buenos Aires, 2003, pp. 63-83].

${ }^{41}$ Michel Foucault, Les mots et les choses, Une archéologie des sciences humaines, Gallimard, Paris, 1966. pp. 265-275 [Traducción al español: Las palabras y las cosas. Una arqueología de las ciencias humanas, $2^{\circ}$ reimpresión, Siglo XXI, Buenos Aires, 2005, pp. 248-258]. Posteriormente, Foucault indicará que la ruptura epistémica que marca el pasaje del clasicismo a la modernidad está vinculada a la emergencia de la población. Foucault, Sécurité, territoire, population, pp. 78-81 [trad. esp., pp. 103-108].

${ }^{42}$ Michel Foucault, Surveiller et punir. Naissance de la prison, Gallimard, Paris, 1975, pp. 222-229 [Traducción al español: Vigilar y castigar. El nacimiento de la prisión, $4^{\circ}$ reimpresión, Siglo XXI, Buenos Aires, 2005, pp. 221-230].

${ }^{43}$ Incluso en Seguridad, territorio, población, Foucault identifica a la biopolítica como el marco de racionalidad de las instituciones médicas. Ver Foucault, Sécurité, territoire, population, pp. 123-124 [trad. esp., pp. 145-146].

${ }^{44}$ Foucault, Naissance de la clinique, pp. 87-106 [trad. esp., pp. 129-153].

${ }^{45}$ Michel Foucault, Les anormaux. Cours au Collège de France (1974-1975), Gallimard, Paris, 1999 [Traducción al español: Los anormales, Curso en el Collège de France, 1974-1975, 3a reimpresión, Fondo de Cultura Económica, Buenos Aires, 2006]. 
esta noción ocupa toda una serie de conferencias brindadas ya desde fines de la década del sesenta ${ }^{46}$.

3. Normalización. La noción de normalización ocupa, de la misma manera, un lugar privilegiado y persistente. Antes incluso de identificar en Surveiller et punir la normalización disciplinaria ${ }^{47}$, Foucault había ya insistido en Histoire de la folie en los efectos generales de normalización vinculados al internamiento ${ }^{48}$; y, en Naissance de la clinique, había señalado la medicalización del conocimiento sobre el hombre a partir de la trasposición de las nociones médicas de lo normal y lo patológico ${ }^{49}$. El poder de normalización es también objeto del curso Les anormaux ${ }^{50}$.

4. Marco de racionalidad: Finalmente, el marco de racionalidad de la biopolítica, articulado en términos de la economía política, ocupa un lugar preeminente. Tanto en Histoire de la folie ${ }^{51}$ como en Naissance de la clinique $e^{52}$, las innovaciones institucionales de la época clásica vienen inscriptas en la parábola que se delinea entre la policía de Estado y el liberalismo económico. La policía de Estado es también recuperada en Surveiller et punir ${ }^{53}$, al tiempo que la economía política es objeto privilegiado de análisis en Les mots et les choses $^{54}$. Finalmente, de expandir el marco de racionalidad al concepto de gubernamentalidad, debería considerarse la definición y el tratamiento de la noción de gobierno en el curso de $1975^{55}$.

En suma, si bien las nociones de biopoder y biopolítica son consideradas en una serie de ocurrencias muy limitadas y, en algún sentido, incidentales; esto no obsta a la incidencia de la biopolítica en la obra de Foucault, desde el momento en que esta noción pone en marcha una serie de preocupaciones que subtienden toda su obra: la población, la medicalización, la normalización y la economía política.

${ }^{46}$ Entre ellas, Michel FoucAult, "Les déviations religieuses et le savoir médical”, en DeFERT y DwALD (dirs.) Dits et écrits II, pp. 652-663; Michel FouCAULT, "Les grands fonctions de la médecine dans notre société", en DefERT y Ewald (dirs.) Dits et écrits II, pp. 1248-1250; Michel FouCaUlt, "Crise de la médecine ou crise de l'antimédecine?", en Defert y Ewald (dirs.), Dits et écrits II, pp. 40-58; Michel FoucAult, "La naissance de la médecine sociale", en DEFERT y EwaLD (dirs.) Dits et écrits II, pp. 207-228; Michel Foucault, "L'incorporation de l'hôpital dans la technologie moderne", en DeFERT y EwaLD (dirs.) Dits et écrits II, pp. 508-521.

${ }^{47}$ Foucault, Surveiller et punir, pp. 180-186 [trad. esp., pp. 182 y 189].

${ }^{48}$ Foucault, Histoire de la folie, pp. 115ss. [trad. esp., t. 1, pp. 132ss.].

${ }^{49}$ Foucault, Naissance de la clinique, pp. 35-36 y 201-203 [trad. esp., pp. 61-62 y 227-280].

${ }^{50}$ FouCAUlt, Les anormaux, pp. 23-24 [trad. esp., pp. 37-60].

${ }^{51}$ Foucault, Histoire de la folie, pp. 70-109 y 477-524. [trad. esp., t. 1, pp. 79-125; t. 2, pp. 66-124].

${ }^{52}$ FouCAULt, Naissance de la clinique, considerar, en este caso, los primeros cinco capítulos.

${ }^{53}$ Foucault, Surveiller et punir, pp. 214-218. [trad. esp., pp. 216-220].

${ }^{54}$ FouCAULt, Les mots et les choses, pp. 265-275 [trad. esp., pp. 248-258].

${ }^{55}$ FouCAUlt, Les anormaux, p. 45 [trad. esp., pp. 55-56]. 